Please do not remove this page

RMIT

UNIVERSITY

\title{
Effects of extremely low frequency electromagnetic fields on electrocardiogram: Analysis with quadratic time-frequency distributions
}

Mahmoud, Seedahmed; Fang, Qiang; Cosic, Irena; Hussain, Zahir

https://researchrepository.rmit.edu.au/esploro/outputs/9921859271001341/filesAndLinks?institution=61RMIT_INST\&index=null

Mahmoud, S., Fang, Q., Cosic, I., \& Hussain, Z. (2005). Effects of extremely low frequency electromagnetic fields on electrocardiogram: Analysis with quadratic time-frequency distributions. Proceedings of the 27th Annual International Conference of the IEEE Engineering in Medicine and Biology Society.

https://doi.org/10.1109/IEMBS.2005.1616545

Published Version: https://doi.org/10.1109/IEMBS.2005.1616545

Repository homepage: https://researchrepository.rmit.edu.au

(C2005 IEEE. Personal use of this material is permitted. However, permission to reprint/republish this material for advertising or promotional purposes or for creating new collective works for resale or redistribution to servers or lists, or to reuse any copyrighted component of this work in other works must be obtained from the IEEE.

Downloaded On 2023/04/26 20:40:00 +1000 


\title{
Effects of Extremely Low Frequency Electromagnetic Fields on Electrocardiogram: Analysis with Quadratic Time-Frequency Distributions
}

\author{
Seedahmed S. Mahmoud, Qiang Fang, Irena Cosic, and Zahir M. Hussain
}

\begin{abstract}
Recently we have studied the effects of extremely low frequency pulsed electromagnetic fields (ELF-PEMF) on the human biosignals. Electrocardiogram (ECG) and electroencephalogram (EEG) of seventeen healthy volunteers before and after the electromagnetic (EMF) exposure were recorded and analyzed. The root mean square (RMS) values of the recoded data were considered as comparison criteria. EEG results were shown that there were small variations in the brain electrical activity before and after exposure. The ECG power level was increased up to $1 \%$ for most of the subjects. In this paper, we further investigate the effects of the ELF-PEMF on the ECG signal using the hyperbolic T-distributions (HTD). This distribution was shown to be suitable for efficient amplitude and instantaneous frequency (IF) estimation of mono- and multicomponent FM signals. In this work, we introduce this distribution to the analysis of ECG signals. The simulation results show that the HTD have a good performance in the analysis of the ECG signals comparing with the Choi-Williams distribution (CWD). Moreover, the results show that there are small shift in the frequency-domain signal before and after EMF exposure.
\end{abstract}

\section{INTRODUCTION}

Over the past few decades, the beneficial therapeutic effects of selected low energy, time varying electromagnetic fields (EMF) have been studied with increasing frequency to treat therapeutically resistant problems of musculoskeletal system [1]. Also, the activities of superoxide dismutase, catalase, and glutathione peroxidase and content of nonprotein thiol groups (reduced glutathione) in blood lymphocytes from patients with rheumatoid arthritis before and during bioresonance therapy (BRT) has been studied in [2]. BRT has been known since the late 1980s but is still poorly studied. It is the therapy with the patient's own oscillations or similar to the body oscillations [3].

Devices that generate EMF are commonly used in sports medicine where it is often economically advantageous to return an injured athlete to good health faster. In these applications clinical devices generating EMFs with fields ranging from a few $\mu$ Tesla to a few milli-Tesla can significantly shorten the healing time of orthopedic injuries [4]. Modern therapy devices can broadly be divided into three frequency bands: radio frequency generators (often operating at 27 $\mathrm{MHz}$ ) using either capacitive or inductive coupling of the energy to the tissue, low frequency magnetic field generators

S. S. Mahmoud, Q. Fang, I. Cosic, and Z. M. Hussain are with the School of Electrical and Computer Engineering, RMIT University, Melbourne, Victoria 3000, Australia mahmoud.seedahmedarmit.edu.au, john. fang@rmit. edu.au,irena.cosic@rmit.edu.au, zahir.hussain@rmit.edu.au.
$(1 \mathrm{~Hz}-10 \mathrm{~Hz})$ and static magnetic field sources [5]. MEDEC bioresonance device is an example of the low frequency class [6].

In the past, ECG signals have been analyzed in either a time-indexed or spectral form. The fact, is that the ECG and all other biological signals belong to the family of multicomponent nonstationary signals. Time-frequency analysis techniques are used as they reveal the multicomponent nature of such signals. However, quadratic time-frequency representations, also known as time-frequency distributions (TFD's), of multicomponent signals suffer from the presence of cross-terms [7]. A subclass of Cohen's Class of TFD's, referred to as the hyperbolic T-distributions (HTD), was presented in [8], [9] and shown to be suitable for efficient amplitude and instantaneous frequency (IF) estimation of multicomponent signals. The hyperbolic time only kernels have proven to be efficient in reducing cross terms while retaining high resolution, with a compromise between these two requirements depending on the selected parameters.

In [10] we investigate the biological effects of the ELFPEMF on the human cardiac and brain signals. The t-test analysis for the EEG bands at the frontal and occipital lobes was indicated that there were no significant difference between the root mean square (RMS) values before and after EMF exposure. In the case of ECG signal, the $95 \%$ confidence interval of the significant result suggested that there was an increased in mean ECG potential. In this paper, we further investigate the effects of the ELF-PEMF on the ECG signal using the time-frequency approach with the optimal smoothing kernels designed in [8]

\section{Materials AND Methods}

This paper studies the effects of the EMF bioresonance therapy on the human heart signal using the hyperbolic Tdistributions. Experimental setup will be discussed in the following subsections.

\section{A. Subjects}

This study was done on seventeen healthy (age of 2035) volunteers. All subjects were hand-right and healthy, without any medical or psychiatric disorders. They were not under any medications that may affect the mental and neural activities.

\section{B. ELF-PEMF Exposure with BRS}

In this work we used the MEDEC Bioresonance (Bioresonance therapy) System (BRS) [10]. This system produces a 
pulsating energy field that stimulates a resonance within the human body. It induces fine currents within the body that are very similar to the body's own currents. It has 28 programs and 8 intensities. Each program has different frequency. In this work we selected program 9 and the intensity of 3 . The pulsed EMF fields for the top, middle and bottom coils of the applicator Mat are $2.33 \mu$ Tesla, $5.235 \mu$ Tesla and 6.45 $\mu$ Tesla, respectively with $16 \mathrm{~Hz}$ operating frequency. The duration of this program is 10 minutes.

\section{ECG Equipment}

In this measurement, Lead-I electrodes was connected to BIOPAC systems, ECG module. The ECG module (ECG 100C) consists of instrumentation amplifier (IA) and a $50 \mathrm{~Hz}$ notch filter. The IA gain was set to 1000 . The ECG data were recorded by using AcqKnowledge software (v.3.7.1, BIOPAC Systems, Inc., CA) in ASCII text files and processed by programs written in Matlab. The sampling rate for ECG was set to 1000 samples/second.

\section{Recording protocols}

In this work, Lead-I was considered in ECG recording [12]. Two stages of recording for this experiment were considered as follows

1) Before exposure: The subject was asked to relax and lie down with his eyes open. The duration of the ECG signal recording was chosen to be 30 seconds.

2) After the exposure: Measurement of thirty seconds duration for the previous components was taken immediately after the ELF-PEMF exposure. The duration of the exposure was 10 minutes.

\section{Time-Frequency Distributions}

As mentioned before, a TFD that provides a good reduction of the cross-terms is needed to make sure that the off-diagonal elements of the TFD matrix of the sources are negligible and so that a diagonal structure can be maintained.

The time-frequency distribution of the analytic signal $z(t)$ associated with the original real signal $s(t)$ can be expressed as follows [8]

$$
\rho(t, f)=\mathcal{F}_{\tau \rightarrow f}\left[\phi(t, \tau) *_{(t)} K_{z}(t, \tau)\right]
$$

where $K_{z}(t, \tau)=z\left(t+\frac{\tau}{2}\right) z\left(t-\frac{\tau}{2}\right)$ is the instantaneous autocorrelation product, $\mathcal{F}$ is the Fourier transform, and $*_{(t)}$ denotes time convolution. The time-lag kernel $\phi(t, \tau)$ completely characterizes the TFD [9].

Wigner-Ville distribution (WVD) and Choi-Williams distribution (CWD) are the most famous TFDs of Cohen's Class [13]. Time-only kernels (kernels of the T-distributions) presented in [8] were shown to be more efficient than their two dimensional counterparts in Cohen's Class in terms of $t-f$ resolution and cross-terms reduction. In this paper, we consider the hyperbolic T-distributions (HTD) for the analysis of the ECG signals. The kernel (the hyperbolic timeonly kernel) for this distribution is given by [8]

$$
\phi(t, \tau)=\phi_{\alpha}(t)=\frac{k_{\alpha}}{\cosh ^{2 \alpha}(t)}
$$

where $\alpha$ is a real positive number and the normalization factor $k_{\alpha}=\int_{-\infty}^{\infty} 1 / \cosh ^{2 \alpha}(t) d t$ which equals $\Gamma(2 \alpha) / 2^{2 \alpha-1} \Gamma^{2}(\alpha)$, where $\Gamma$ is the gamma function. Hence, the TFD of the analytic signal $z(t)$ is given by

$$
\rho(t, f)=\mathcal{F}_{\tau \rightarrow f}\left[\frac{k_{\alpha}}{\cosh ^{2 \alpha}(t)} *(t) K_{z}(t, \tau)\right] .
$$

\section{RESUlTS AND DiscusSiON}

In this section, the results of seventeen subjects artifactfree Lead-I ECG data were analyzed. These data were recorded for 30 seconds. Two techniques of analysis were considered for the ECG data. These techniques are: the time interval analysis and the time-frequency analysis.

\section{A. Time Intervals Analysis}

The time intervals of PR, RT, QT, and RR before and after exposure were measured and compared (Fig. 1). The RR interval, the period of rhythmic electrical activity of the heart, is the reciprocal of heart rate. The rhythmic pacemaker of the sinoatrial node is principally responsible for the regular beating of the heart, but the pacemaker action potential, which occurs near the start of the P-wave, is too small to be seen in the ECG. The PR interval represents the time for conduction of electrical activity from atria to ventricles, the P-wave and Q-wave or R-wave of the ECG indicating the onset of cardiac-cell depolarization in atria and ventricles, respectively. The QT interval is an index of the duration of the ventricular action potential, with the T-wave indicating ventricular repolarization [11], [14].

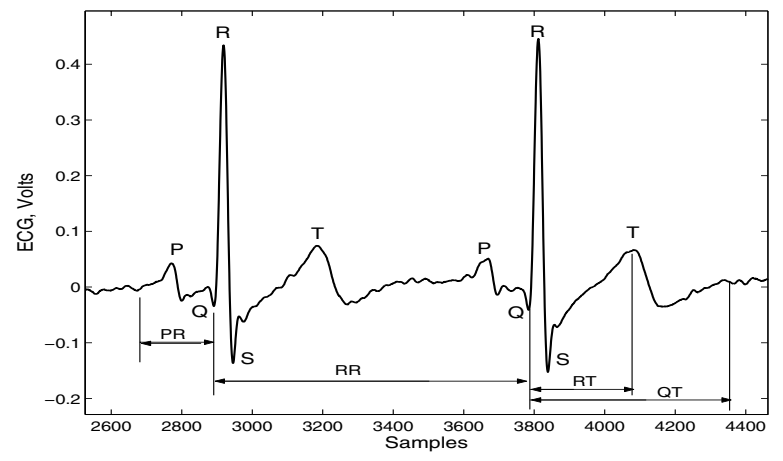

Fig. 1. ECG time intervals measured before and after EMF exposure.

Fig. 2 shows the deviation for PR, RT, QT and RR ECG intervals before and after the exposure. From this figure, we observe that there IS no significants change in most of these intervals. This indicates that the ELF-PEMF didn't significantly affect the electrical activity of cardiac tissue where these events originated. However, for the RR interval there is small change which may contribute to the heart rate value (see Fig. 2). 


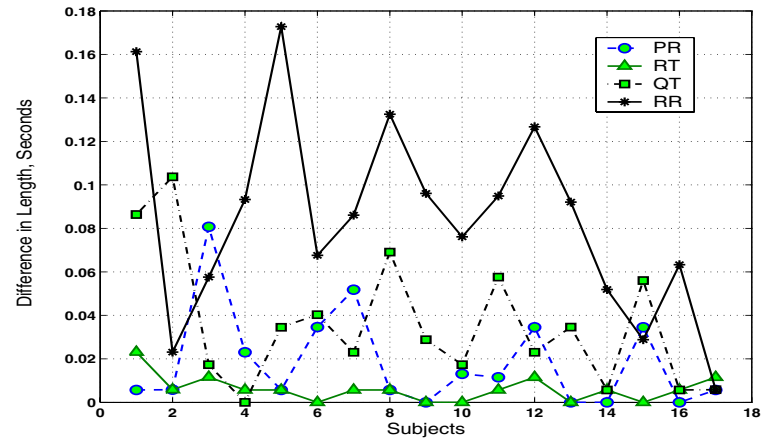

Fig. 2. The difference in length for PR, RT, QT and RR intervals, before and after ELF-PEMF exposure.

\section{B. Time-Frequency Analysis}

All ECG signals were down-sampled to 50 samples/second. The mean of the ECG data was calculated and then subtracted from the ECG signal in order to remove any DC offset. Further, 15 seconds ECG signals were converted by Hilbert transform into their analytical forms and processed using T-distributions. In all numerical simulation, we set the value of $\alpha=0.05$ for the HTD.

For space limitations, we do not show the time-frequency distributions of all subjects. Based on Fig. 2, we will provide the time-frequency analysis for subjects 1,5 and 12 . Other subjects' analysis results observed the same results as in the previous subjects (frequency-shift before and after EMF exposure).

Figs. 3, 5 and 7 show the time-frequency distribution of the ECG signals for subject 1, 5, and 12, respectively. While, Figs. 4, 6 and 8 show the frequency components of the ECG signals for subject 1,5 , and 12 at the time instants $t=10$ seconds, respectively. From these figures we observe that there is small shift in the frequency-domain signal before and after EMF exposure. This can be clearly observed and related to Fig. 2, where the interval RR was experienced small change.
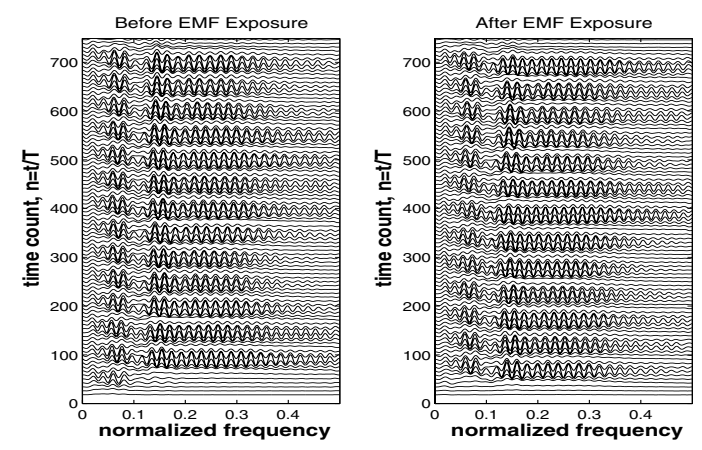

Fig. 3. Hyperbolic T-distribution of the ECG signal for subject1 (with $\alpha=0.05$, sampling frequency $=50$ samples $/$ second, and signal length $=750$ samples).

Fig. 9 shows a comparison between the CWD and the HTD as related to resolution and cross-term reduction at the

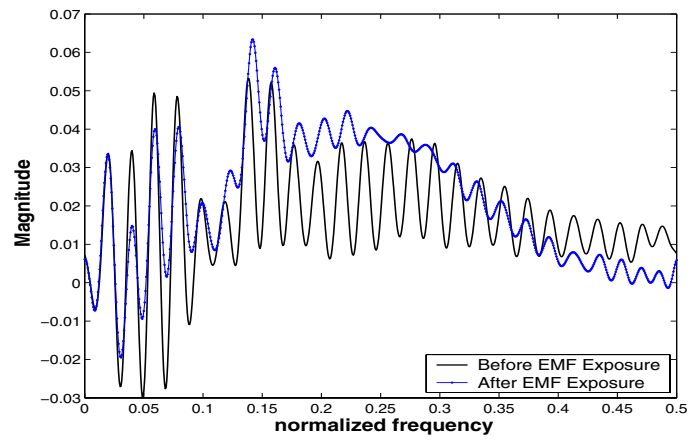

Fig. 4. Frequency components of the ECG signal for subject-1 at the time instants $t=10$ seconds.
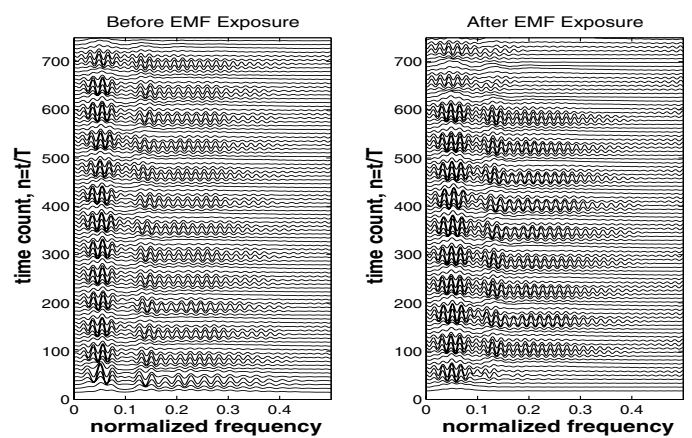

Fig. 5. Hyperbolic T-distribution of the ECG signal for subject5 (with $\alpha=0.05$, sampling frequency $=50$ samples/second, and signal length $=750$ samples $)$.

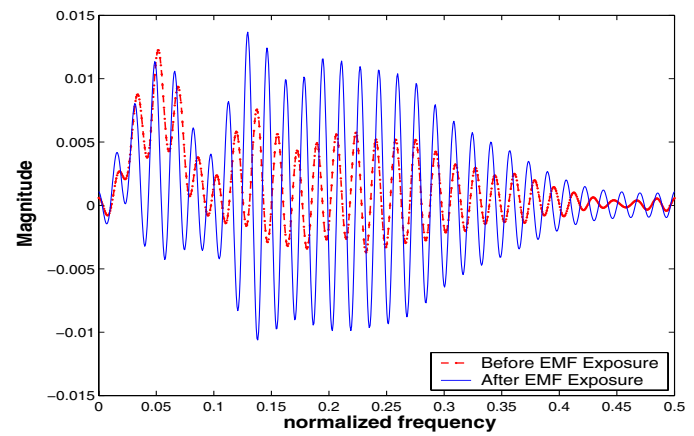

Fig. 6. Frequency components of the ECG signal for subject-5 at the time instants $t=10$ seconds.

time count $n=500$ (time instants $t=10$ seconds) for subject 12 after the EMF exposure.

\section{CONCLUSION}

This paper provide a further investigation to the effects of the ELF-PEMF on the ECG signal using the hyperbolic Tdistributions. The MEDEC Bioresonance therapeutic system was used to generate the ELF-PEMF. In the ECG time interval analysis, the results showed that there is small change in the RR interval. However, there are no significants change for other intervals. This indicates that the only interval affected by the EMF exposure on the heart activity is the RR interval 

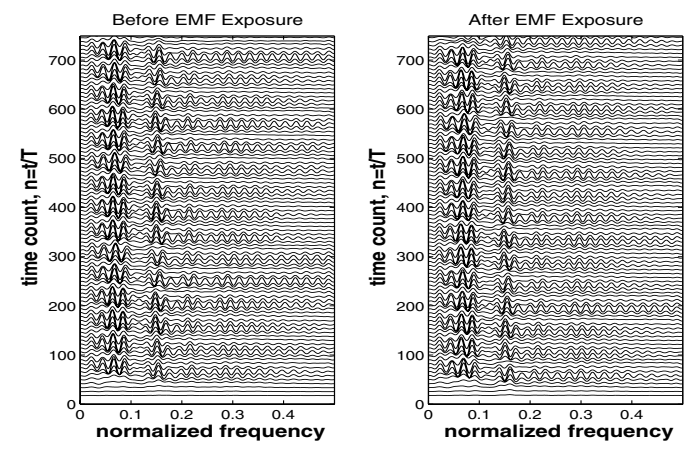

Fig. 7. Hyperbolic T-distribution of the ECG signal for subject12 (with $\alpha=0.05$, sampling frequency $=50$ samples/second, and signal length $=750$ samples).

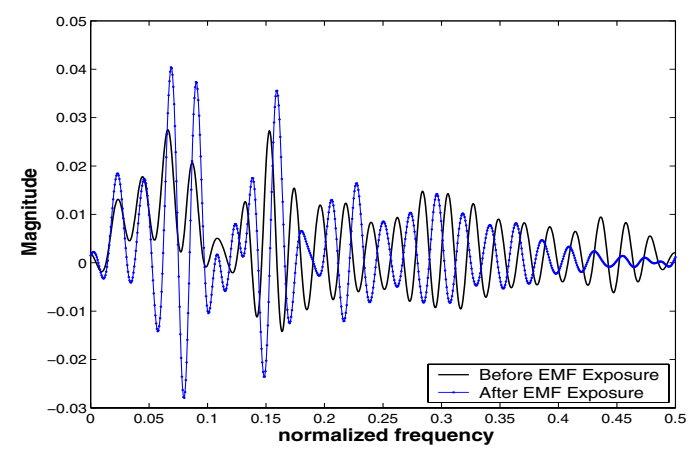

Fig. 8. Frequency components of the ECG signal for subject-1 at the time instants $t=10$ seconds.

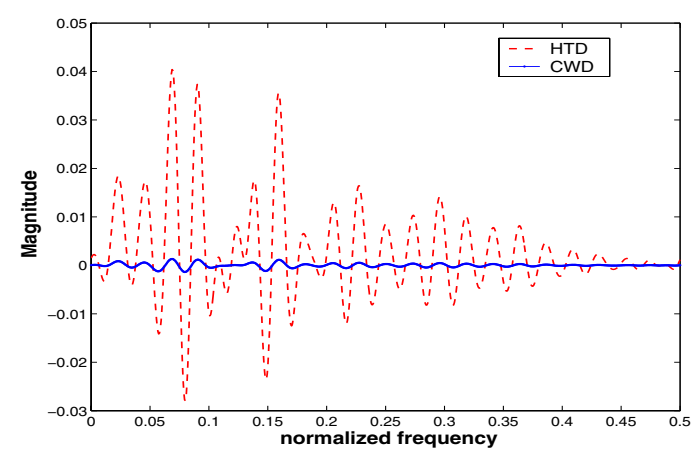

Fig. 9. Comparison between the CWD and the HTD as related to resolution and cross-term reduction at the time count $n=500$ for subject 12 after the EMF exposure (with $\alpha=0.05$ for HTD, $\alpha=19$ for CWD, sampling frequency $=50$ samples/second, and signal length $=750$ samples).

(the reciprocal of heart rate). In the time-frequency analysis, the results show that there is small shift in the frequencydomain signal before and after EMF exposure. This is due to the variation that was occurred in the RR interval.

\section{ACKNOWLEDGMENTS}

The authors would like to thank MEDEC Ltd, Australia, for providing the Bioresonance device that was used in this work. This research is supported by a RIF grant from RMIT University.

\section{REFERENCES}

[1] K. Chang, W. H. Chang, M. Wu, and C. Shih, "Effects of Different Intensities of Extremely Low Frequency Pulsed Electromagnetic Fields on Formation of Osteoclast-like Cells," Biolectromagnetics, vol. 24, pp. 431-439, 2003.

[2] B. I. Islamov, R. M. Balabanova, V. A. Funtikov, Y. V. Gotovskii, and E. E. Meizerov, "Effects of Bioresonance Therapy on Antioxidant System in Lymphocytes in Patients with rheumatoid arthritis," Bulletin of Experimental Biology and Medicine, Biophysics and Biochemistry, No. 3, pp. 248-250, 2002.

[3] A. Fedorowski, A. Steciwko, and J. Rabczynski, "Low-Frequency Electromagnetic Stimulation May Lead to Regression of Morris Hepatoma in Buffalo Rats," The Journal of Alternative and Complementary Medicine, Vol. 10, No. 2, pp. 251260, 2004.

[4] G. Rein, "Bioinformation Within the Biofield: Beyond Bioelectromagnetics," The Journal of Alternative and Complementary Medicine, vol. 10, No. 1, pp. 59 - 68, 2004.

[5] A. T. Barker, "Electricity, magnetism and the body: some uses and abuses," Engineering Science and Education Journal, no. 12, pp. 249-256, 1993.

[6] Medec Inc, "MEDEC Bioresonance System Manual," Medec international, ABN 68079053268, Western Australia, Australia.

[7] B. Boashash, "Time-frequency signal analysis," in Advances in Spectrum Estimation, S. Haykin, Ed. Englewood Cliffs, NJ: PrenticeHall,1991.

[8] Zahir M. Hussain and Boualem Boashash, "Adaptive instantaneous frequency estimation of multi-component FM signals using quadratic time-frequency distributions," IEEE Transactions on Signal Processing, vol. 50, no. 8, pp. 1866 -1876, August 2002.

[9] Z. M. Hussain and B. Boashash, "Design of time-frequency distributions for amplitude and IF estimation of multicomponent signals," The Sixth International Symposium on Signal Processing and Its Applications (ISSPA2001), 13-16 Aug. 2001.

[10] Seedahmed S. Mahmoud, Qiang Fang, and Irena Cosic, "Effects of Extremely Low Frequency Pulsed Electromagnetic Fields on Biosignals," Submitted to Journal of Bioelectromagnetics.

[11] R. L. Seaman and J. R. Jauchem, "Rat Electrocardiogram During Acute Exposure to Synchronized Burst of Ultra-Wideband Pulses," IEEE Transactions on Plasma Science, vol. 32, no. 4, pp. 1644 1652, Aug. 2004.

[12] J. J. Carr and J. M. Brown, Intoduction to Biomedical Equipement Technology, Prentice-Hall, 1998.

[13] L. Cohen, "Time-Frequency Analysis," Prentice-Hall, Englewood Cliffs, NJ, 1995.

[14] A. M. Katz, Physiology of the Heart, 2nd ed. New York: Raven, 1992. 